\begin{tabular}{|l|l|c|c|}
\hline Eiszeitalter u. Gegenwart & 35 & $\begin{array}{c}147-160 \\
5 \mathrm{fig} .\end{array}$ & Hannover 1985 \\
\hline
\end{tabular}

\title{
Analyse de variations minéralogiques par surfaces de tendance dans les tephra du volcan El Chichón (Chiapas, Mexique)
}

\author{
Jean-Paul Donnay \& Etienne Juvigné *)
}

Three dimensional model, heavy minerals, light minerals, statistical extension, petrographic analysis, volcanic ash, computer program, particle shape, specific gravity.

Mexico, Chiapas (Volcano El Chichon)

R é s u m é : Des résultats d'analyses minéralogiques des tephra émises par le volcan El Chichón en mars-avril 1982 ont été soumis à une analyse statistique multivariée. Des variations spatiales du contenu minéralogique ont été cartographiées par surfaces de tendance. Des cartes de résidus de régressions ont été dréssées. Une sélection progressive des minéraux au cours du transport du nuage de poussière est fermement établie; elle dépend de la forme et du poids spécifique des grains. La turbulence exceptionnellement élevée dans les régions proches du volcan perturbe cette sélection.

[Verbreitungsmodell von mineralogischen Variationen in den Aschen vom Vulkan El Chichón (Chiapas, Mexico)]

$\mathrm{K}$ u r z f a s s u n g : Im Verbreitungsgebiet der vom Vulkan El Chichón im Zeitraum März bis April 1982 geförderten Eruptiva wurden 74 Ascheproben entnommen. Die Leicht- und Schwermineralführung der entnommenen Proben wurde quantitativ bestimmt. Mittels eines Computersprogramms wurde für geeignete Minerale ein statistisch gesichertes Verbreitungsmodell erstellt. Dabei zeigt sich, daß die Minerale nach Kornform und spezifischen Gewicht entsprechend die Entfernung vom Ausbruchspunkt sortiert werden. Die Abweichungen der Werte zu den theoretisch berechneten sind in der Nähe des Eruptionszentrums am größten. Das Verbreitungsmuster der Minerale wird in diesem Raum von den Strömungsverhältnissen in der Stratosphäre abgeleitet.

[Trend Surfaces of Mineralogical Variations within the Tephra Lobe of El Chichón Volcano (Chiapas, Mexico)]

A b s r a c t : Quantitative analysis of minerals from 74 tephra samples of the MarchApril 1982 eruption of El Chichón have been submitted to multivariate statistical techniques.

*) Adresse des auteurs: J.-P. Donnay, Assistant, Séminaire de Géographie, Université de Liège, place du XX Août 7, B 4000 Liège, Belgique.

E. Juvignê, Chercheur Qualifié au Fonds National de la Recherche Scientifique, Laboratoire de Géomorphologie et de Géologie du Quaternaire, Université de Liège, place du XX Août 7 , B 4000 Liège, Belgique. 
Variations in mineralogical content have been mapped by trend surfaces and maps of residual values have been drawn. A progressive sorting of the minerals during transport in the atmosphere has been firmly demonstrated; this sorting depends on both specific gravity and shape of the grains. Exceptionally high turbulence in the vicinity of the volcano disturbs the sorting.

\section{Introduction}

En analysant quelques échantillons des retombées du volcan Quizapu (Chili), LARSSON (1935) fut probablement le premier à démontrer, par des résultats semiquantitatifs, l'existence d'une différentiation minéralogique intervenant au cours du transport dans l'atmosphère d'un nuage de poussière volcanique.

Juvigné \& SHIPLEY (1983) étudiant la retombée du 18 mai 1980 du volcan Mount Saint Helens (Washington - U.S. A.) ont mis en évidence des variations quantitatives du contenu en minéraux denses dans la partie distale du lobe.

JUVIGNÉ (1983) a étendu ce type de recherche aux trois retombées de mars-avril 1982 du volcan El Chichón (Chiapas-Mexique). Il compare, à l'aide de régressions linéaires simples, la dispersion relative de 9 types de minéraux.

Dans ces deux dernières recherches, l'analyse statistique des données a été réalisée uniquement le long d'un transect à travers chacune des zones de dispersion, et des cartes de variations du contenu minéralogique des tephra ont été réalisées de façon essentiellement empirique.

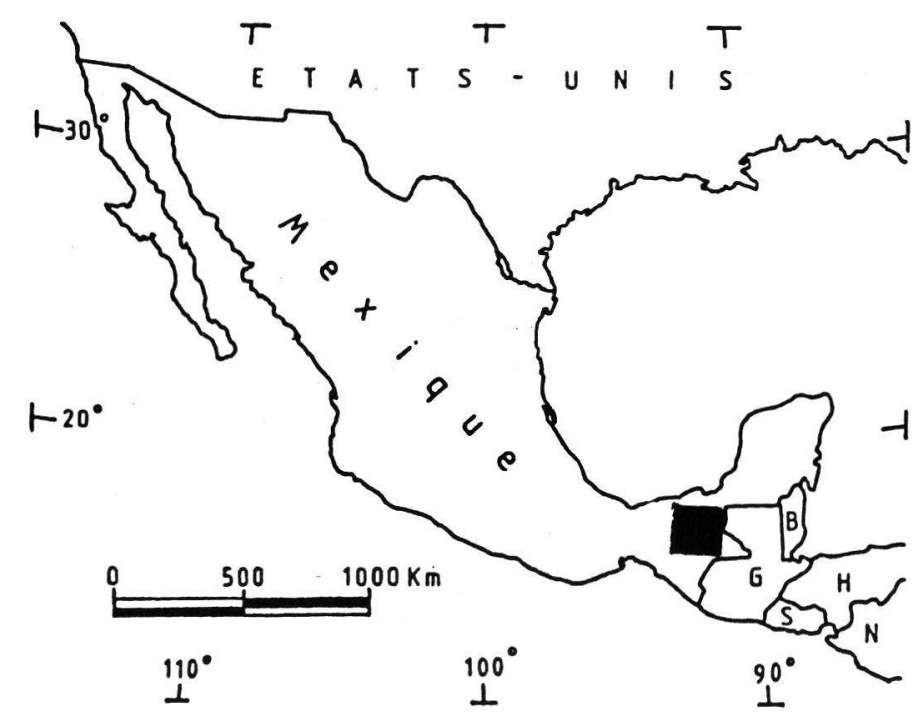

Fig. 1: Localisation de la région étudiée.

Le carré noir correspond à la surface de toutes les cartes publiées dans cet article. 
Dans le présent article, les résultats des comptages, réalisés par JuviGNÉ (1983) sur 74 échantillons prélevés dans le lobe de retombées du volcan El Chichón, ont servi de base à une analyse multivariée, traitée et informatisée par Donnay.

La région étudiée est localisée sur la figure 1, où le carré noir correspond à la surface des différentes cartes présentées dans cet article. Les signes figurant sur les cartes de la figure 5 renseignent sur la localisation des 74 échantillons étudiés.

\section{Trajectoire dans l'atmosphère d'un nuage de poussière volcanique et principe de l'analyse statistique}

Dans le cas d'éruptions explosives de magmas acides, un nuage de poussière est éjecté jusque dans la stratosphère où il se détend brutalement puis est pris en charge par un jet-stream qui oriente nettement sa distribution.

Les particules solides du nuage tendent alors à retomber à la surface de la terre et la vitesse de chute de chaque grain est fonction, notamment, de sa taille, son poids spécifique et sa forme. Ces facteurs provoquent nécessairement un triage au sein du nuage et un classement des particules dans la couche de tephra déposée à la surface du sol.

Une telle sélection peut être mise en évidence par un ajustement tridimensionnel, communément appelé surface de tendance, qui met en relation, d'une part, la concentration en minéraux denses: $Z$, et, d'autre part, les coordonnées $\mathrm{X}$ et $\mathrm{Y}$ des sites retenus dans l'échantillon.

$$
\begin{aligned}
Z & =\sum_{\mathrm{i}} \sum_{j} \text { bij } \cdot \mathrm{X}^{\mathrm{i}} \cdot \mathrm{Y}^{\mathrm{j}}+\mathrm{e} \\
\text { où }: Z & =\text { valeur de la surface } \\
\mathrm{e} & =\text { variable aléatoire avec certaines propriétés } \\
\mathrm{X}, \mathrm{Y} & =\text { coordonnées planes } \\
\text { i et } \mathrm{j} & =\text { puissances successives des coordonnées } \mathrm{X} \text { et } \mathrm{Y}
\end{aligned}
$$

La cartographie des résultats d'une telle analyse constitue un outil descriptif intéressant, sous la forme d'une carte par isolignes lissant les accidents locaux au profit d'une tendance générale.

D'autre part, l'équation de l'ajustement optimal et l'analyse des résidus de la régression sont susceptibles de nous éclairer tant sur le modèle de répartition envisagé que sur les conditions locales de dépôt.

\section{Les conditions d'application}

L'application de la régression polynomiale a été imaginée après la récolte et le traitement des échantillons, de sorte qu'aucun plan de sondage spécifique n'a pu être dicté. Ainsi, les sites de prélèvements sont irrégulièrement espacés, ce qui rend difficile 
l'emploi de polynômes orthogonaux. Nous avons dès lors résolu la régression polynomiale de manière classique, selon la procédure de régression linéaire multiple et ce, quelles que soient les corrélations observées entre les variables.

Enfin, il est clair que cette application semble particulièrement bien adaptée à l'usage d'un espace vectoriel, défini en coordonnées polaires. Cependant, nous nous limiterons ici à une analyse dans le système de coordonnées cartésiennes. En effet, l'étude de ce type de tephra devrait, à plus long terme, conduire à l'élaboration d'un modèle général susceptible d'éclairer des données tephrostratigraphiques relatives à des éruptions anciennes. Or dans ce contexte, l'association de différents sites sédimentaires constitue le problème essentiel. Le lieu d'émission des poussières étant généralement inconnu, la définition d'un espace vectoriel est rendue impossible.

\section{Le programme de traitement des données}

Nous avons écrit un programme réalisant à la fois le calcul de la régression polynomiale et la cartographie de la surface de tendance associée, selon le degré défini par l'utilisateur.

Le logiciel, écrit en Pascal U.C.S.D., tourne sur une configuration centrée sur un micro-ordinateur (Apple ][) et la cartographie est réalisée sur une imprimante Epson $\mathrm{RX}-80$, en mode graphique »bit-image «.

La partie du programme consacrée à la résolution de la régression est une traduction et une adaptation d'un programme publié par Baillargeon (BAILARGEON \& RaINVILLE 1979). Le programme peut être utilisé aux seules fins de la régression multiple traditionelle.

Lorsque le programme est utilisé pour ajuster une surface de tendance, les données nécessaires sont:

- les coordonnées $\mathrm{X}$ et $\mathrm{Y}$

- les valeurs de la variable dépendante $Z$

- le degré du polynôme

Les variables nécessaires aux calculs des polynômes de degré supérieur à l'unité sont créées par le programme.

Les paramètres utiles à la cartographie sont:

- le niveau de base des isolignes NB

- l'équidistance EQUI

- l'échelle de prise de coordonnées

Treize trames à grains fins sont prévues, depuis et y compris le »blanc « jusqu'au noircissement complet. Si des valeurs inférieures au niveau de base se présentent, elles sont incluses à la première classe, tandis que des valeurs supérieures à la limite maximum prévue $\left[\left(13^{*} \mathrm{EQUI}\right)+\mathrm{NB}\right]$, sont ramenées à la dernière classe. Les trames extrêmes sont donc associées à des classes ouvertes telles que:

première classe: $\hat{Z}<\mathrm{NB}+\mathrm{EQUI}$

dernière classe: $\hat{Z}>\mathrm{NB}+12{ }^{*} \mathrm{EQUI}$ 
Les extrema de la distribution des valeurs de $\hat{Z}$ sont repris en légende.

La connaissance de l'échelle utilisée lors de l'enregistrement des coordonnées, permet au programme de calculer l'échelle de reproduction qui est mentionnée sur le listing sous la forme d'une échelle graphique.

Le temps nécessaire aux calculs et à l'impression des tableaux statistiques et de la carte est bien sûr fonction du nombre de données et du degré du polynôme choisi. Dans le cas de cette application ( 74 données), le traitement complet de la surface de degré trois prend quelque 18 minutes. Signalons, en outre, que l'impression des tableaux statistiques est optionelle. Cela permet la réalisation de plusieurs cartes consécutives, selon des paramètres divers, en un temps minimum.

Enfin, une fois l'équation de la surface de tendance calculée, elle peut être introduite à l'entrée d'un programme de visualisation à trois dimensions. Une vue isométrique paramétrable de la surface peut, ainsi, être réalisée.

\section{L'analyse et les résultats}

Tous les renseignements utiles, relatifs à la localisation, aux méthodes de prélèvement et de traitement des échantillons, ainsi que toutes les données quantitatives propres à leur contenu minéralogique sont disponibles dans l'article de JUvIGNÉ (1983).

Les coordonnées des 74 sites de prélèvement, localisés sur la figure 1 dans l'article de Juvigné (1983), sont obtenues par digitalisation avec une précision qui est, sur le terrain, de l'ordre du kilomètre. L'origine du système de coordonnées a été fixée au sudouest de la région étudiée.

\subsection{Détermination du meilleur ajustement}

Des surfaces des quatre premiers degrés ont été testées sur les différents jeux de données. Au terme de cette recherche, il est apparu que la surface de troisième degré constitue l'ajustement optimal aux données disponibles.

A titre d'exemple, nous rapportons ici les résultats essentiels de l'étude comparative effectuée sur les données relatives au contenu global en minéraux denses.

La variable $Z$ représente la part de minéraux denses mesurée en pourcent du poids total (500 milligrammes par échanillon). Elle se distribue de la façon suivante (en \%):

$$
\begin{aligned}
& \text { minimum: } 0.5 \text { maximum: } 51.0 \\
& \text { moyenne: } 15.6 \quad \text { écart-t.: } 9.8
\end{aligned}
$$

L'analyse a porté sur les quatre premiers degrés du polynôme. Pour juger de l'efficacité de l'adjonction de termes supplémentaires, nous avons utilisé un test $\mathrm{F}$ construit sur l'apport de variance expliquée propre au degré considéré (UNWIN 1975), soit: 


$$
\mathrm{F}=\frac{(\operatorname{Ve}(\mathrm{n})-\operatorname{Ve}(\mathrm{n}-1)) / \mathrm{d} 11}{(100-\operatorname{Ve}(\mathrm{n})) / \mathrm{d} 12}
$$

$$
\begin{aligned}
\text { où: } \operatorname{Ve}(\mathrm{n}) & =\text { variance expliquée par la surface de degré } \mathrm{n}(\text { en } \%) \\
\operatorname{Ve}(\mathrm{n}-1) & =\text { variance expliquée par la surface de degré } \mathrm{n}-1 \text { (en \%) } \\
\mathrm{d} 11 & =\text { degrés de liberté associés aux termes additionnels } \\
\mathrm{d} 12 & =\text { degrés de liberté associés aux résidus }
\end{aligned}
$$

La signification de la surface de premier degré est testée par un test $\mathrm{F}$ traditionnel qui apparaît comme un cas particulier du test mentionné ci-dessus.

Tab. 1.: Analyse de variance des surfaces de tendance degrés 1 à 3 (contenu global en minéraux denses)

Source de variation

$\mathrm{d} 1$

$\mathrm{Ve}$

F

Surface linéaire

2

36.63

Résidus à la surface linéaire

71

63.37

Surface quadratique

3

35.83

Résidus à la surface quadratique

68

27.54

Surface cubique

4

14.56

Résidus à la surface cubique

64

12.96

20.52

29.49

18.00

La surface de premier degré rend compte de quelque $37 \%$ de la variation de la variable $Z$. Le plan de régression est alors incliné vers l'est avec une très légère inflexion vers le sud-est. Néanmoins le coefficient relatif à la variable Y n'est pas significativement différent de zéro (au seuil de $0.01 \%$ ).

L'apport de variance expliquée par les termes de la surface quadratique est assez considérable (36\%). Ils déterminent l'apparition d'un sommet sur la surface, centré sur le site du volcan. D'autre part, l'influence des nouveaux coefficients significatifs conduit à l'obtention d'ellipses concentriques. Le grand axe de ces ellipses (gradient de plus faible pente) est orienté ouest-est.

La surface cubique produit une augmentation significative du coefficient de détermination (15\%). L'allure générale de la surface est assez similaire à celle obtenue au degré précédent. Néanmoins, les termes additionnels de la surface cubique provoquent au moins une modification intéressante à noter. On constate que la longueur des axes de 


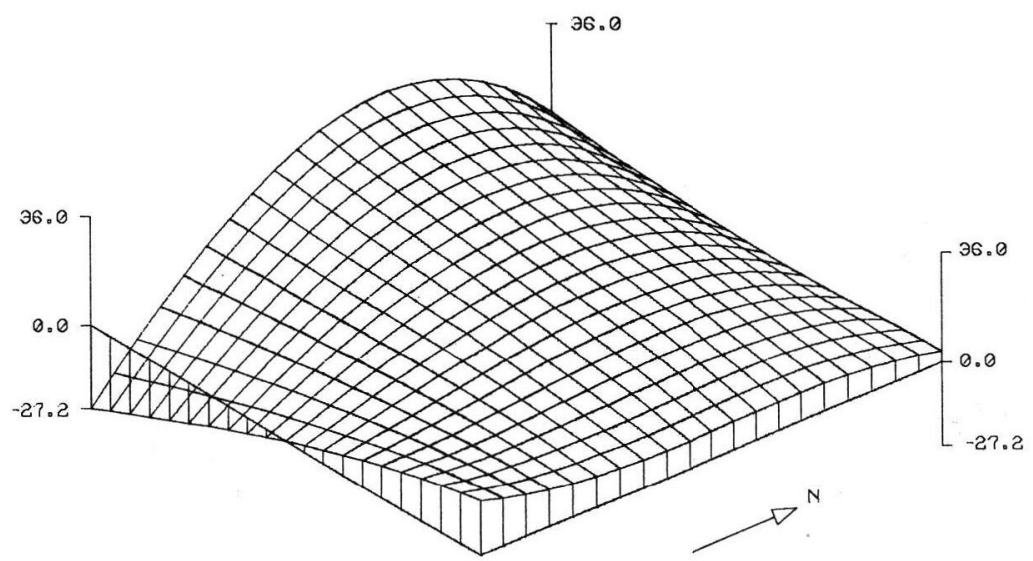

Fig. 2: Visualisation tri-dimensionelle de la surface de régression de degré 3 relative au contenu global en minéraux denses des retombées de mars-avril 1982 du volcan El Chichón;

les valeurs des $Z$ sont exprimées en pourcent du poids total de l'échantillon. La figure 3 constitue une représentation bi-dimensionelle du même phénomène.

toutes les demi-ellipses est plus courte qu'à l'étape précédente. Le gradient de pente est donc plus affirmé et cela, dans toutes les directions. La surface couverte par les minéraux denses en est réduite d'autant. La visualisation tri-dimensionnelle de cette surface de régression du troisième degré est illustrée à la figure 2 .

L'effet de marge, qui semble générer un second sommet sur la surface dans le coin sud-est de la carte, est sans signification propre. Rappelons que cette partie du territoire n'a pu être échantillonnée.

La contribution de la surface quartique n'est pas significative, de sorte que la surface de troisième degré constitue l'ajustement optimal à ce jeu de données (variance expliquée cumulée: $87.04 \%$ ). L'équation est de la forme suivante:

$$
\begin{aligned}
\hat{Z}= & b(0)+b(1) \cdot X+b(2) \cdot Y+b(3) \cdot X^{2}+b(4) \cdot X \cdot Y+b(5) \cdot Y^{2}+b(6) \cdot X^{3} \\
& +b(7) \cdot X^{2} \cdot Y+b(8) \cdot X \cdot Y^{2}+b(9) \cdot Y^{3}
\end{aligned}
$$

Nous reprenons dans le tableau 2 les dix coefficients de régression relatifs, respectivement, au contenu global en minéraux denses, à la part des clinopyroxènes et à celle des hornblendes. Dans ces deux derniers cas, la variable est mesurée en pourcent du nombre total de grains de minéraux denses transparents présents dans l'échantillon, et l'ajustement d'une surface du troisième degré rend compte de quelque $60 \%$ de la variance totale.

Les trop faibles parts d'apatite ( 1 à $9 \%)$ et de sphène ( 0 à $2 \%)$ conduisent à des résultats non significatifs dès le premier ajustement (exemple: apatite, $\mathrm{r}$ carré $=0.05$ ). 


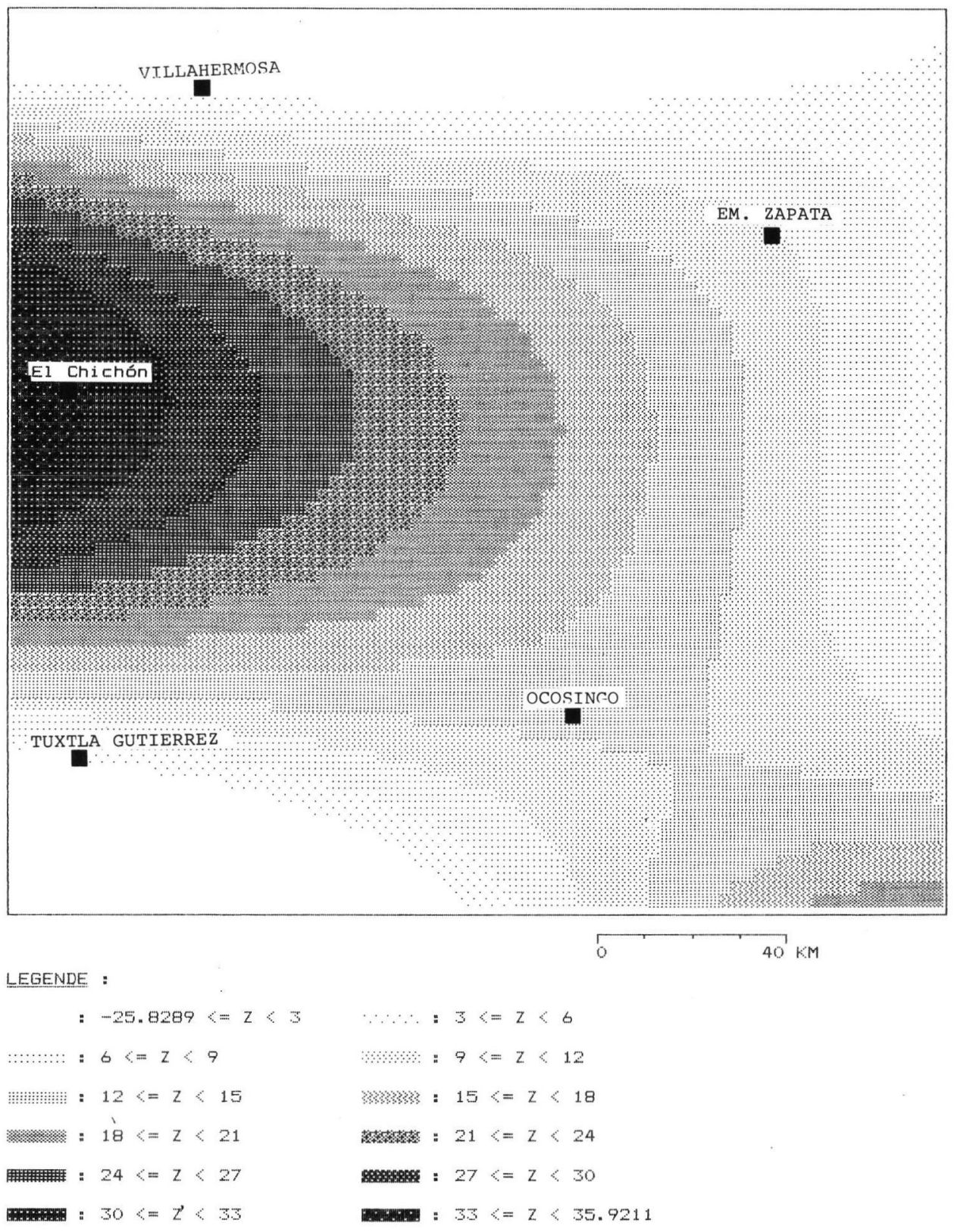

Fig. 3: Variation de la part de l'ensemble des minéraux denses (Z) dans les retombées du volcan El Chichón; les valeurs de $Z$ sont exprimées en pourcent du poids total de l'échantillon.

Les 74 sites d'échantillonnage sont localisés sur la figure 5 . 
Tab. 2: Coefficients de régression des trois analyses

\begin{tabular}{lccc}
\hline & Minéraux denses & Clinopyroxènes & Hornblende \\
\hline & & & \\
\hline $\mathrm{b}(0)$ & -612.358302 & -147.864965 & 212.117004 \\
$\mathrm{~b}(1)$ & 4.93901794 & 0.985342767 & -0.87984952 \\
$\mathrm{~b}(2)$ & 6.66751555 & 2.20442408 & -1.66954172 \\
$\mathrm{~b}(3)$ & $-4.36086535 \mathrm{E}-4$ & $7.70981635 \mathrm{E}-4$ & $6.96628093 \mathrm{E}-4$ \\
$\mathrm{~b}(4)$ & -0.0648839129 & -0.0167474828 & 0.0131973314 \\
$\mathrm{~b}(5)$ & $-4.08414577 \mathrm{E}-3$ & $-4.34265425 \mathrm{E}-3$ & $2.17784522 \mathrm{E}-3$ \\
$\mathrm{~b}(6)$ & $-1.07938101 \mathrm{E}-5$ & $1.40330672 \mathrm{E}-5$ & $-1.30104979 \mathrm{E}-5$ \\
$\mathrm{~b}(7)$ & $3.1768434 \mathrm{E}-5$ & $-3.46437358 \mathrm{E}-5$ & $2.23986885 \mathrm{E}-5$ \\
$\mathrm{~b}(8)$ & $1.77269519 \mathrm{E}-4$ & $8.05066775 \mathrm{E}-5$ & $-5.99712341 \mathrm{E}-5$ \\
$\mathrm{~b}(9)$ & $-6.44336942 \mathrm{E}-5$ & $-1.79353051 \mathrm{E}-5$ & $-1.7582619 \mathrm{E}-5$ \\
& & & \\
\hline
\end{tabular}

\subsection{Les surfaces de tendance}

Les figures 3 et 4 représentent les surfaces de troisième degré ajustées aux trois jeux de données. Avec les équations qui les accompagnent, elles constituent des documents de référence pour les comparaisons de variations de même type, relatives à d'autres retombées passées ou à venir.

Ces cartes mettent en évidence une direction préférentielle de dispersion dirigée vers l'est, et font bien ressortir les variations spatiales des sélections qui sont intervenues dans les nuages de poussières. Ces variations sont, en effet, plus adoucies le long du grand axe que dans la direction perpendiculaire. Cette différence est particulièrement nette sur la carte du contenu global en minéraux denses.

Cette disposition atteste clairement que la densité des particules est un facteur déterminant pour les sélections qui s'opèrent dans les nuages de poussières volcaniques. Ceux-ci, au cours de leur progression, tendent donc à perdre plus rapidement leurs minéraux denses que les légers et les verres. Ceci indique également la tendance d'un nuage à devenir essentiellement vitreux au cours de ses révolutions successives dans la stratosphère.

En ce qui concerne les pourcentages de clinopyroxènes et de hornblendes, il est nécessaire de rappeler que les chiffres sont toujours relatifs au total des minéraux denses présents dans l'échantillon. La faible représentation des minéraux denses, autres que les clinopyroxènes et hornblendes, fait que les parts de ces derniers sont quasiment complémentaires. Il faut donc s'attendre à une inversion de la répartition des concentrations relatives, selon que l'on examine l'un ou l'autre de ces minéraux (figure 4). Ces variations de concentrations relatives, tout intéressantes qu'elles sont pour les comparaisons ultérieures avec d'autres sites volcaniques, ne fournissent pas de renseignement sur la répartition absolue des minéraux en présence. 


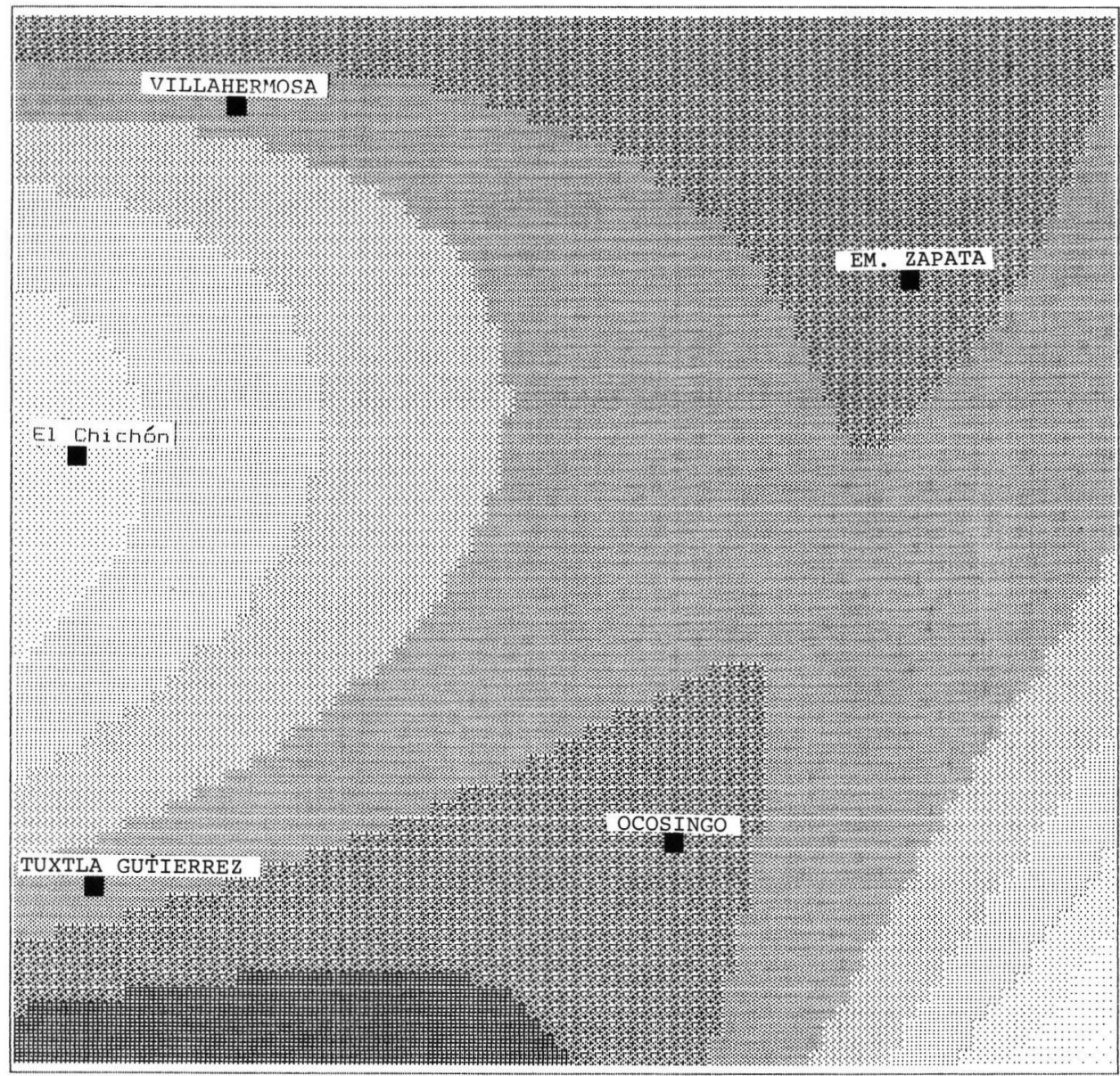

\section{Hornblende}

LEGENDE :

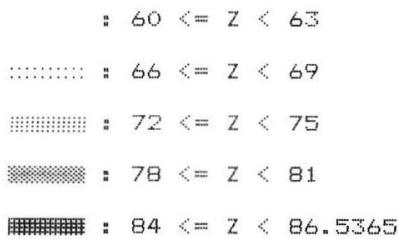

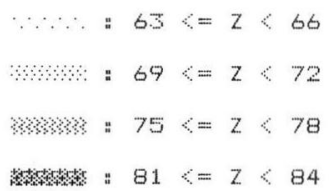

Fig. 4: Variation des parts de 2 minéraux $(\mathrm{Z})$ au sein de l'association des minéraux denses transparents dans les retombées du volcan El Chichón; les valeurs des $Z$ sont exprimées en pourcent du nombre de grains de minéraux denses transparents dans l'échantillon. Les 74 sites d'échantillonnage sont localisés sur la figure 5. 


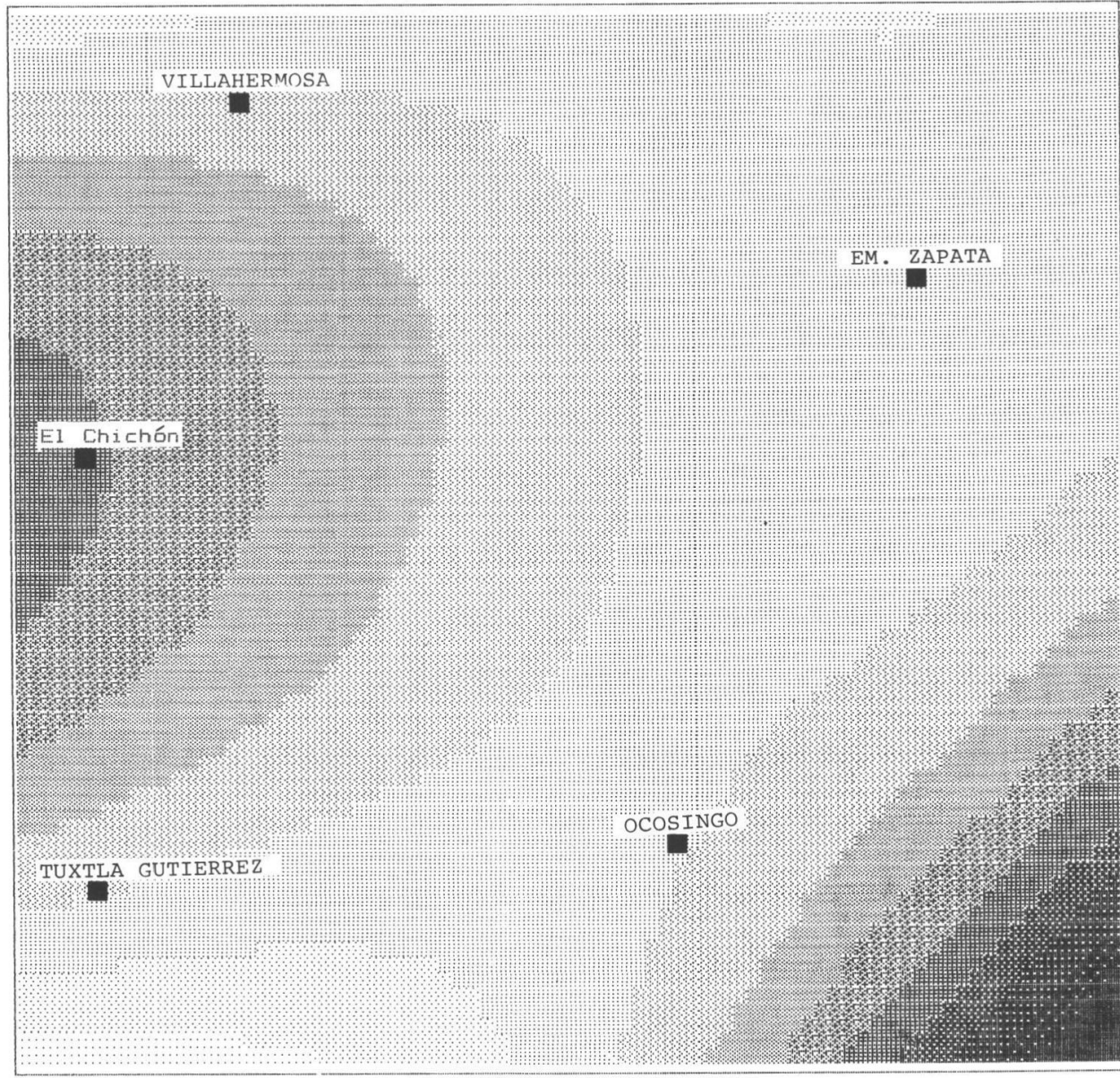

\section{Clinopyroxène}

\section{LEGENDE :}

$: 0<=2<3$

: $6<=7<9$

HU1! $12<=2<15$

\% $\times 18<z<21$

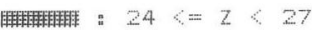

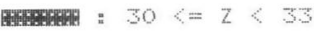

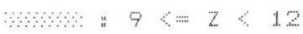

ห४४ : $15=2<18$

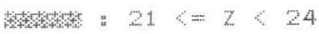

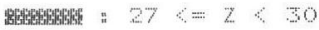

36 $=z<41.9455$

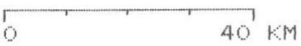



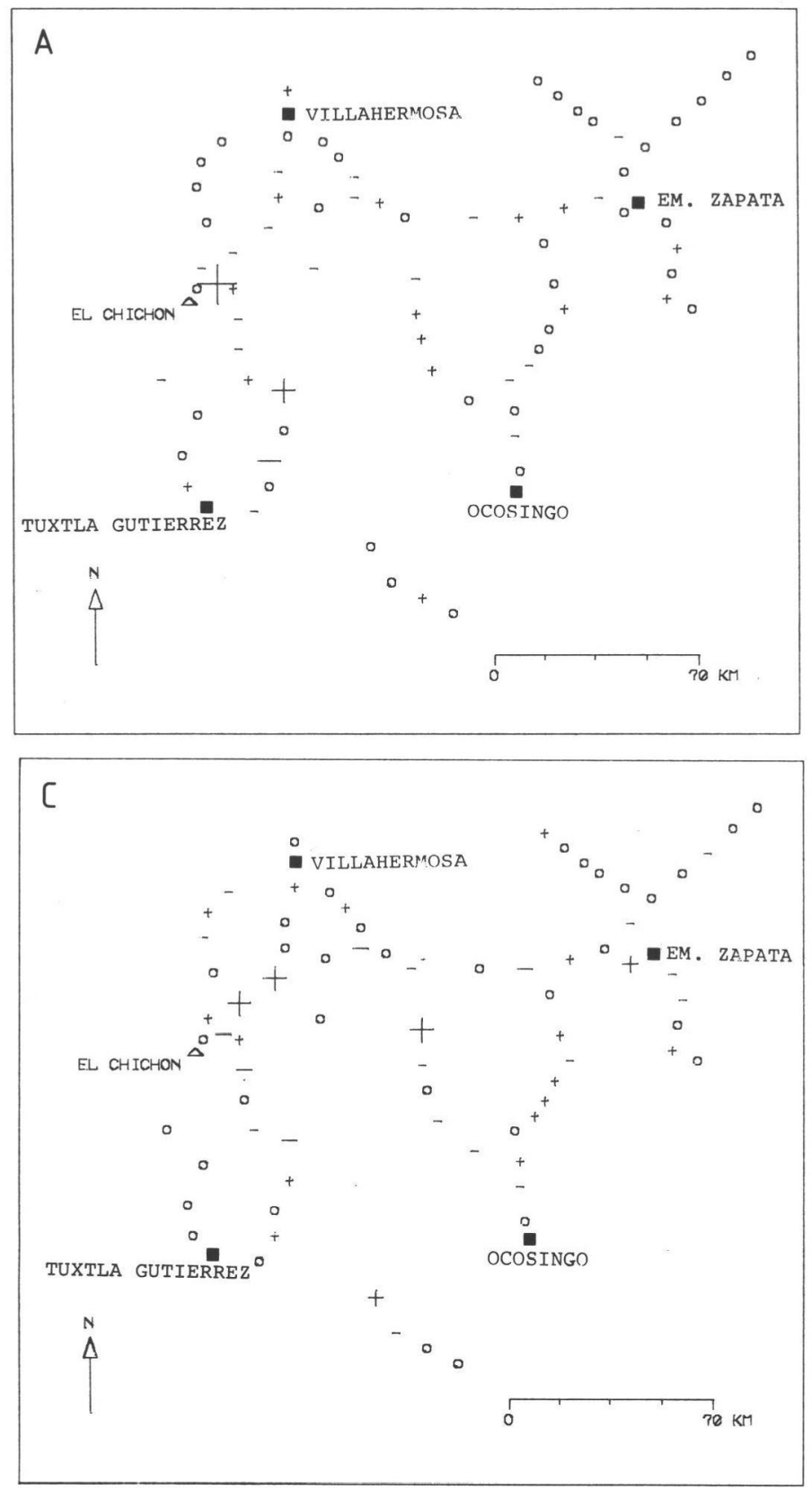

Fig. 5: Résidus standardisés des analyses de régression arrêtées au 3ème degré pour:

A. le contenu global en minéraux denses $(s=3.81)$

B. le part des clinopyroxènes $(s=3.58)$

C. la part des hornblendes $(s=3.50)$

$s=$ erreur-standard de l'estimation. 


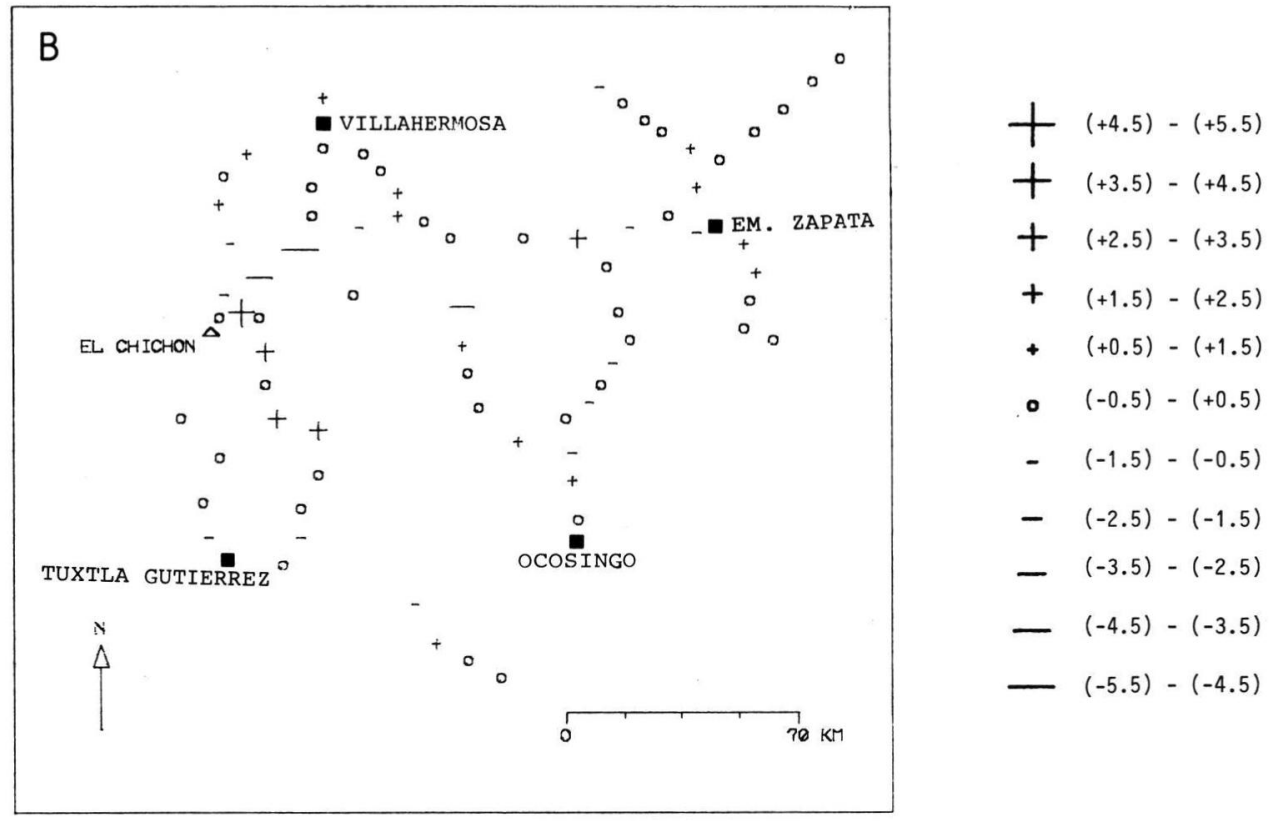

En l'absence d'informations en chiffres absolus, ces deux analyses s'interprètent uniquement en termes de rapport clinopyroxène/hornblende, et l'explication du processus de dépôt demeure hypothétique. La couronne de concentration maximum de hornblende coincide-t-elle avec un dépôt maximum de ce minéral, ou indique-t-elle seulement la marge de dépôt du clinopyroxène? L'amplitude de variation des concentrations de hornblende conduit plutôt à retenir la seconde solution. Un processus différentiel de dépôt, caractérisé par des maxima successifs selon les minéraux, ne semble donc pas vérifié.

\subsection{Les résidus des régressions}

Des cartes de résidus standardisés ont été établies respectivement pour le contenu global en minéraux denses, la part des clinopyroxènes et celle des hornblendes. L'erreurstandard de l'estimation et les valeurs résiduelles extrêmes sont présentées au tableau 3.

Tab. 3: Caractéristiques des résidus de régression (surface de degré 3)

\begin{tabular}{lccc}
\hline & $\begin{array}{c}\text { Erreur- } \\
\text { Standard }\end{array}$ & $\begin{array}{c}\text { Valeurs (en \%) } \\
\text { Minima et Maxima }\end{array}$ \\
\hline Contenu global en minéraux denses & 3.81 & -9 & +18 \\
Clinopyroxènes & 3.58 & -11 & +9 \\
Hornblendes & 3.80 & -7 & +11 \\
\hline
\end{tabular}


La répartition géographique des résidus est représentée sur les cartes de la figure 5 .

Si aucune tendance ne semble se manifester, la plupart des résidus, pris en valeur absolue, sont concentrés dans un cercle d'une soixantaine de kilomètres de rayon autour du volcan. Les environs du site du volcan sont soumis, lors de l'éruption, à une turbulence importante. Ce phénomène est susceptible d'expliquer le classement hétéroclite des sédiments dont rendent compte les résidus de régression. Des renseignements plus précis, notamment sur les conditions locales de dépôt, sont cependant nécessaires pour étayer cette hypothèse.

\section{Conclusion}

Les variations spatiales du contenu minéralogique des tephra du volcan El Chichón ont été mises en évidence par une analyse de régression polynomiale multiple: l'analyse des surfaces de tendance. Elle montre que les minéraux retombent d'autant plus rapidement qu'ils sont denses et massifs. Les variations observées sont nettement moins brutales selon la direction principale de dispersion que perpendiculairement à celle-ci.

Ces observations sont à mettre en relation avec des phénomènes de sélection qui interviennent au cours du transport du nuage de poussière dans la stratosphère.

\section{Remerciements}

Quelques-uns des échantillons analysés dans ce travail nous ont été fournis par le Dr. R. Coutino (U.N.A.M.); nous le remercions très vivement.

\section{References}

Baillargeon, G. \& Rainville, J. (1979): Statistique appliquée, 3: Régression multiple. - (2ème édition), Les Editions SMG; Trois-Rivières.

Cliff, A. D., Haggett, P., Ord, J. K., Bassett, K. \& Davies, R. (1975): Elements of Spatial Structure. - Cambridge (Cambridge University Press).

JuviGNÉ, E. (1983): Les variations minéralogiques dans les retombées de 1982 du volcan El Chichón (Chiapas, Mexique) et leur intérêt pour la téphrostratigraphie. - Ann. Soc. Géol. Belg., 106: 311-325; Liège.

- \& SHIPLeY, S. (1983): Distribution of the heavy minerals in the downwind tephra lobe of the May 18, 1980 eruption of the Mount Saint Helens (Washington, U.S.A). - Eiszeitalter u. Gegenwart, 33: 1-7; Hannover.

Larsson, W. (1935): Vulkanische Asche vom Ausbruch des chilessischen Vulkans Quizapu (1932) in Argentina. - Geol. Inst. Upsala Bull., 26: 27-52; Upsala.

UNwIN, D. (1975): An introduction to trend surface analysis., - CATMOG, Institute of British Geographers, 5. Geo Abstract Ltd.; Norwich. 